\title{
Primary Tumor Location Implications on Biological Therapy in Metastatic Colorectal Cancer
}

\author{
Marwan Fakih \\ Medical Oncology, Gastrointestinal Oncology, City of Hope Comprehensive Cancer Center, California, US
}

DOI: https://doi.org/10.17925/OHR.2017.13.01.30

\begin{abstract}
A nti-epidermal growth factor receptors (anti-EGFRs) have been clinically proven to improve overall survival (OS) when combined with chemotherapy in the settings of extended RAS wild-type metastatic colorectal cancers. In addition, randomized phase III studies have confirmed the superiority of anti-EGFR therapy over bevacizumab in the front-line treatment of extended RAS wild-type metastatic colorectal cancer when it comes to response rate, and in select studies, OS. Recent updates from these clinical trials project convincing evidence that tumor sidedness is yet another predictive marker for response to anti-EGFR therapy. While left colonic RAS wild-type metastatic colorectal cancers derive major clinical benefit from anti-EGFR versus bevacizumab therapy, bevacizumab appears to have clear advantage in RAS wild-type metastatic right-sided tumors. In this review, we summarize recent reports from key clinical trials and their impact on day-to-day clinical practice.
\end{abstract}

Keywords

Sidedness, colorectal cancer, epidermal growth factor, metastatic disease, cetuximab, panitumumab, bevacizumab, chemotherapy

Disclosure: Marwan Fakih has nothing to declare in relation to this article. This study involves a review of the literature and did not involve any studies with human or animal subjects performed by any of the authors. No funding was received for the publication of this article.

Authorship: All named authors meet the International Committee of Medical Journal Editors (ICMJE) criteria for authorship for this manuscript, take responsibility for the integrity of the work as a whole and have given final approval for the version to be published.

open Access: This article is published under the Creative Commons Attribution Noncommercial License, which permits any non-commercial use, distribution, adaptation and reproduction provided the original author(s) and source are given appropriate credit.

Received: February 20, 2017

Accepted: April 7, 2017

Citation: Oncology \& Hematology Review 2017;13(1):30-3

Corresponding Author: Marwan Fakih, City of Hope Comprehensive Cancer Center, 1500 E Duarte Rd, Duarte, CA 91010, US. E: mfakih@coh.org
Several studies have shown a survival advantage with the addition of anti-epidermal growth factor receptor (anti-EGFR) therapy to chemotherapy in molecularly selected patients with advanced colorectal cancer. The Panitumumab Randomized trial in combination with chemotherapy for Metastatic colorectal cancer to determine Efficacy (PRIME) clinical trial investigated the addition of panitumumab to folinic acid, fluorouracil, and oxaliplatin (FOLFOX) in patients with KRAS exon-2 wild-type metastatic colorectal cancer. ${ }^{1}$ The addition of panitumumab to chemotherapy improved overall survival (OS) by $17 \%$ and translated in a statistically significant increase in median OS from 19.7 to 23.9 months. ${ }^{1}$ Further analysis confirmed a lack of benefit from panitumumab in exon-3 and exon-4 KRAS mutations and exon 2, 3, and 4 NRAS mutations, therefore limiting panitumumab recommendations to extended RAS wild-type metastatic colorectal cancer. In this subgroup of patients, panitumumab improved the OS by $22 \%$, extending the median OS from 20.2 to 26.0 months. ${ }^{2}$ Similar findings have been reported in the Cetuximab combined with iRinotecan in first-line therapY for metastatic colorectal cancer (CRYSTAL) trial, a randomized phase III clinical trial comparing folinic acid, fluorouracil, and irinotecan (FOLFIRI) to FOLFIRI plus cetuximab in the first-line treatment of KRAS wild-type metastatic colorectal cancer. The addition of cetuximab to FOLFIRI improved OS by $20 \%$ and was associated with a significant improvement in median survival from 20.0 to 23.5 months. ${ }^{3}$ Further analysis confirmed a lack of benefit from non-exon 2 KRAS and exons 2, 3, and 4 NRAS mutations. The addition of cetuximab to FOLFIRI in RAS wild-type metastatic colorectal cancer improved OS by $31 \%{ }^{4}$ These studies, and other supporting evidence from second-line (and beyond) clinical trials support the benefit from antiEGFR therapy in extended RAS wild-type colorectal cancer. ${ }^{5}$ Additional evidence suggests that BRAF mutations, in addition to their poor prognostication, are also associated with lack of clinical benefit from anti-EGFR therapy. ${ }^{6}$ Recent studies, however, suggest that in addition to RAS and BRAF status, tumor sidedness has a considerable impact on metastatic colorectal cancer outcome, specifically with anti-EGFR therapy. In this review, we review pertinent literature on right and left colon cancer and its impact on anti-EGFR therapy recommendations in patients with advanced colorectal cancer.

\section{Biological differences between right and left colon}

The right and left colons are embryologically distinct. The right colon is derived from the midgut and consists of the cecum, ascending colon, and the proximal two-thirds of the transverse colon. The left colon is derived from the hindgut and extends from the end of the transverse colon cancer to the rectum. ${ }^{7}$ Indeed, the biological differences between the right and the left colon have been shown to exist at the molecular level—as confirmed by extensive gene expression studies on colonic tissue from human embryos and adults. ${ }^{8}$ Therefore, it is not surprising that distinct molecular differences have been seen between right and left colorectal cancers across different genomic data sets. Missaglia et 
al. reported on the biology of colorectal cancers from the PETACC-3 and the TCGA data sets. ${ }^{9}$ Right colonic tumors were more likely to be microsatellite instable (MSI) or MSI-like, associated with a RAS or a BRAF signature, have a serrated pathway, and to have a JAK-STAT gene signature. Left colonic tumors were more likely to be associated with WNT and MYC pathways activation, to have beta catenin activation, and to be associated with EGFR and HER2 upregulation. Even upon limiting the analysis to patients with microsatellite stability (MSS), KRAS wild-type, and BRAF wild-type tumors, left colonic tumors were associated with EGFR upregulation, which was lacking in right colonic tumors. These major variations between left and right colonic cancers, particularly at the EGFR expression and activation level, provide support to the subsequent clinical data that suggest a lack of anti-EGFR activity in right colonic tumors, irrespective of RAS status. It is likely that left colonic tumors, in contrast to right colonic tumors, are dependent on an overactive EGFR-pathway, making them more susceptible to EGFR blockade as a therapeutic strategy.

\section{Prognostic implications of right and left colon cancers}

Several studies have shown that the os of colorectal cancer patients is negatively impacted by right sidedness. However, considerable debate remains around the interaction between tumor location and stage vis-à-vis OS. Both Weiss et al., and Meguid et al. reported on sidedness and outcome based on analyses of the Surveillance, Epidemiology, and End Results (SEER) data base. ${ }^{10,11}$ Both studies showed a worse outcome in patients with right colonic tumors, particularly those with stage III disease. Other populationbased studies reported on the OS of stage I-III colon cancers and generated conflicting results as to the impact of sidedness on outcome. ${ }^{12-14}$ Recent analysis of prospective clinical trials sheds additional light on the impact of sidedness in earlier stage disease, specifically in stage III colon cancer. The PETACC-3 clinical trial randomized 3,045 patients with stage III disease to receive fluorouracil (5-FU) and leucovorin (LV) versus FOLFIRI in the adjuvant setting. No difference in OS was noted between the two treatment arms. Patients with right colon tumors had similar rates of relapse as patients with left colon. However, once a patient relapsed, the OS was clearly impacted by sidedness, with an inferior outcome being noted in rightsided cancers. ${ }^{9}$ Similarly, a combined analysis of two randomized phase III clinical trials (Vioxx in Colorectal Cancer Therapy [VICTOR] and Adjuvant capecitabine plus bevacizumab versus capecitabine alone in patients with colorectal cancer [QUASAR-2]) failed to show a difference in relapse free survival (RFS) between right and left colons while confirming a significantly worse outcome in survival postrelapse in right versus left colon cancers. ${ }^{15}$ The above studies suggest that the impact of sidedness on outcome is more relevant following the development of metastatic disease, reflecting a possible difference in biology and response to therapy following the development of distant metastases. Such hypothesis is clearly supported by a recent meta-analysis of first-line metastatic disease clinical trials which showed a consistent and significant worsening in OS in metastatic colorectal cancer originating in the right colon. ${ }^{16}$ Since the poor os in metastatic right colon cancer has been reported in studies that precede the use of anti-EGFR therapy, one cannot attribute the divergence in outcome to a difference in anti-EGFR response alone.

\section{Anti-epidermal growth factor recepter therapy and sidedness}

There is mounting clinical evidence that anti-EGFR response can be predicted by tumor sidedness in patients with RAS wild-type metastatic
Table 1: Clinical outcome among KRAS wild-type metastatic colorectal cancers by tumor location on C0.17 clinical trial

\begin{tabular}{|l|l|l|l|l|}
\hline \multicolumn{2}{|c|}{} & \multicolumn{2}{c|}{$\begin{array}{c}\text { Right Colon } \\
\text { KRAS Wild-Type }\end{array}$} & \multicolumn{2}{c|}{$\begin{array}{c}\text { Left Colon } \\
\text { KRAS Wild-Type }\end{array}$} \\
\cline { 1 - 5 } & Cmab & BSC $(n=27)$ & Cmab $(n=60)$ & BSC $(n=45)$ \\
\hline PFS & $1.9 \mathrm{~m}$ & $1.9 \mathrm{~m}$ & $5.4 \mathrm{~m}^{*}$ & $1.8 \mathrm{~m}$ \\
\hline OS & $6.2 \mathrm{~m}$ & $3.5 \mathrm{~m}$ & $10.1 \mathrm{~m}^{*}$ & $4.8 \mathrm{~m}$ \\
\hline
\end{tabular}

$\mathrm{Cmab}=$ cetuximab; $B S C$ = best supportive care; $P F S=$ progression free survival; OS = overall survival; $m=$ months; ${ }^{*}$ (denotes statistical significance; $\left.p<0.05\right)$.

colorectal cancer. In this section, we review supportive clinical evidence originating from retrospective patient-series and from the retrospective analysis of prospectively conducted clinical trials.

\section{Chemotherapy-refractory colorectal cancer}

Moretto et al. recently reported on the outcome of 75 RAS wild-type patients with irinotecan-resistant colorectal cancer. ${ }^{17}$ Only 14 out of 75 patients had right colon cancer and were treated with anti-EGFR alone (10 patients) or anti-EGFR + irinotecan (four patients). None of the right-sided colon cancer patients experienced a response and only two had stable disease as best response. Sixty-one patients with left colon cancer were treated with antiEGFR monotherapy (75\%) or anti-EGFR plus irinotecan. Forty percent of patients with left-sided tumors experienced an objective response and an additional 39\% had stable disease. Significant differences were noted between the right and the left colon in terms of progression free survival (PFS) (2.3 versus 6.6 months) and OS (6 versus 15.3 months). These data suggest limited efficacy for anti-EGFR in the treatment of RAS wild-type refractory right colorectal cancer. In an earlier study, de Roock reported on 203 patients with RAS and BRAF wild-type irinotecan-refractory metastatic colorectal cancer who were treated with cetuximab plus irinotecan. ${ }^{18}$ Patients with right colon cancer $(n=45)$ experienced a much shorter time to progression (hazard ratio $[H R]=1.48, p=0.02$ ) than patients with left colon cancer ( $n=158$ ). Last, a retrospective analysis of the pivotal C0.17 clinical trial randomizing patients with chemotherapy-resistant metastatic colon cancer to cetuximab or best supportive care reported on the outcome of KRAS wild-type right and left colon cancers (Table 1). ${ }^{19}$ Benefits in PFS and OS were limited to patients with left-sided tumors. Only 56 patients with right colon cancer were subject to analysis in this study, and despite a lack of a survival advantage in right colon tumors, the median OS was 6.2 versus 3.5 months in favor of patients receiving cetuximab. ${ }^{19}$ Taken in total, the above studies suggest minimal to no benefit with anti-EGFR therapy in right colon cancer. It is important to note that the sample size limitations do not allow for the exclusion of a possible small survival advantage from anti-EGFR therapy or the possibility of substantial benefit from anti-EGFR therapy in a select, but small, population with right colonic tumor and RAS wild-type tumors. The benefits in left colonic RAS wild-type tumors on the other hand are clear and the clinical benefits are substantial.

\section{First- and second-line metastatic colorectal cancer}

In a retrospective study, Wang et al. described the outcome of 416 patients receiving chemotherapy plus cetuximab or chemotherapy alone in the first-line and second-line treatment of metastatic colorectal cancer. ${ }^{20}$ Efficacy outcomes from response rate (RR) to OS were improved in patients receiving chemotherapy and cetuximab over chemotherapy in first- and second-line setting in left colon tumors. No benefits were seen 
Table 2: Clinical outcome among RAS wild-type metastatic colorectal cancers by tumor location across select randomized first- and second-line phase III clinical trials

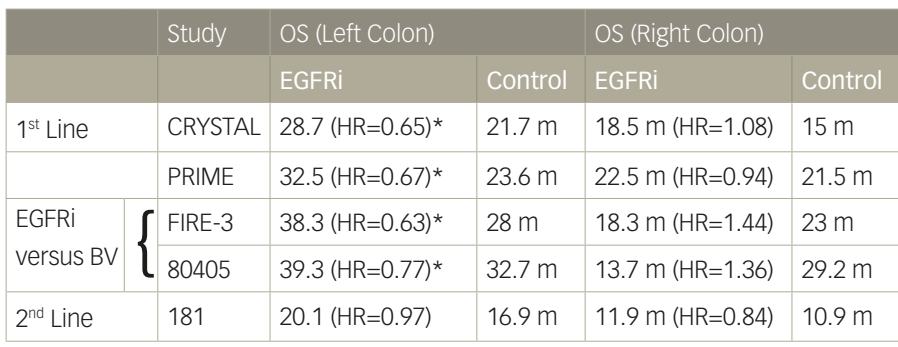

OS = overall survival; EGFRI $=$ epidermal growth factor inhibitor (cetuximab or panitumumab): $B V=$ bevacizumab; $H R$ = hazard ratio; $m=$ months; * (denotes statistical significance: $p<0.05$ ).

with cetuximab in right colonic tumors. More conclusive evidence comes from retrospective analyses of first-line randomized clinical trials. Five randomized first- and second-line phase III studies have now published or presented on the outcome of patients with RAS wild-type metastatic colon cancer in the setting of anti-EGFR therapy by primary tumor location. The CRYSTAL and PRIME clinical trials reported on the outcome of first-line chemotherapy with or without cetuximab and panitumumab, respectively. On the CRYSTAL trial, adding cetuximab to chemotherapy significantly improved RR (72.5\% versus 40.6\%), PFS (12 versus 8.9 months), and OS (28.7 versus 21.7 months) over chemotherapy alone only in patients with left colon tumor (Table 2). ${ }^{21}$ Similar superior results were noted in left colonic tumors on the PRIME clinical trial, with RR (70.3\% versus 54.8\%), PFS (12.9 versus 9.3 months), and OS (32.5 versus 23.6 months) (Table 2). ${ }^{22}$ No benefit, but no detrimental impact, was noted when anti-EGFR therapy was added to chemotherapy in right colonic tumors. These clinically relevant improvements strongly support the incorporation of either cetuximab or panitumumab in the front-line treatment of RAS wild-type left colon with metastatic disease. Similar trends have been demonstrated in the 181 second-line study of FOLFIRI with our without panitumumab when limiting the analysis to RAS wild-type colon cancer (Table 2). ${ }^{22}$ Patients with left colon cancer experienced a significant improvement in RR $(50.7 \%$ versus $13.5 \%$ ), with a trend of improvement in PFS (8 versus 6.6 months) and OS (20.1 versus 16.9 months). No benefits were noted in any efficacy measures with panitumumab in right colonic tumors.

Given the prevalent use of anti-angiogenic therapy in the first- and second-line treatment of metastatic colorectal cancer, a head-to-head comparison of anti-EGFR therapy to anti-angiogenesis therapy is a more relevant question in today's day-to-day practice. The FIRE-3 clinical trial randomized 592 patients with KRAS wild-type metastatic colorectal cancer to FOLFIRI plus bevacizumab versus FOLFIRI plus cetuximab. ${ }^{23}$ While this study failed its primary endpoint of RR, significant improvements in OS were noted in favor of cetuximab that were accentuated further in the RAS wild-type population. In a post hoc analysis of FIRE-3 outcome by colon side, a significant improvement in OS was limited to the left colon tumor patient population (38.3 versus 28 months; $\mathrm{HR}=0.63, \mathrm{p}=0.02$ ). ${ }^{21}$ Patients with right colon cancer had a trend toward a worsened OS outcome with cetuximab versus bevacizumab ( 18.3 versus 23 months; HR=1.44, $\mathrm{p}=0.28){ }^{21}$ Similar trends were noted recently on Cancer and Leukemia Group B (CALGB 80405), a randomized clinical trial of chemotherapy (FOLFOX or FOLFIRI) with cetuximab versus bevacizumab in the first-line treatment of KRAS wild-type metastatic colorectal cancer. ${ }^{24}$ No difference
Figure 1: First-line treatment algorithm in good performance patients with metastatic colorectal cancer

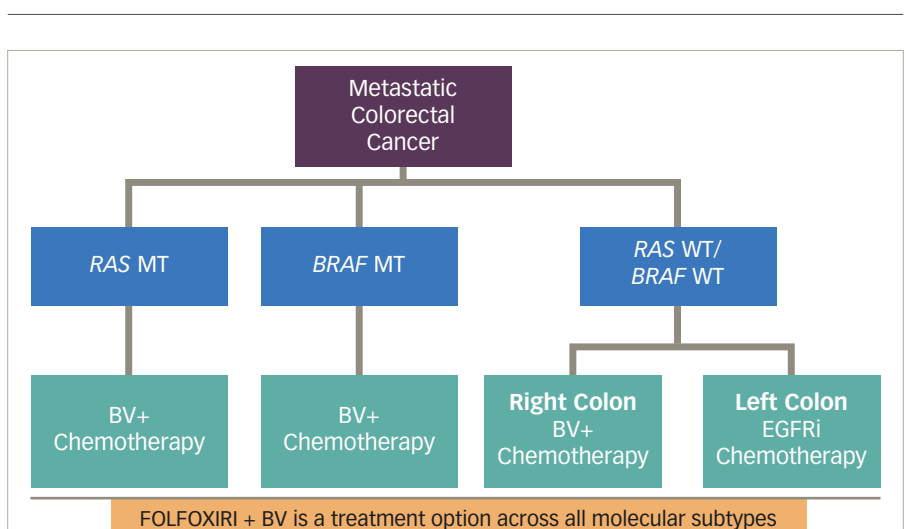

FOLFOXIRI + BV is a treatment option across all molecular subtypes

$M T=$ mutated $: W T=$ wild-type: $B V=$ bevacizumab: $E G F R i=$ epidermal growth factor receptor inhibitor (cetuximab or panitumumab); FOLFOXIRI = folinic acid, fluorouracil, oxaliplatin, irinotecan.

in PFS or OS was noted between arms, although cetuximab was associated with improved RR. ${ }^{24}$ However, a significant biologic interaction was noted by sidedness for both PFS and OS. ${ }^{25}$ Significant improvements in OS were noted in favor of cetuximab (39.3 versus 32.7 months; HR=0.77, $\mathrm{p}=0.04$ ) in the left colonic tumors while cetuximab was associated with a worse outcome for both PFS (7.5 versus 10.2 months; $H R=1.64, p=0.006$ ) and OS (13.7 versus 29.2 months; $H R=1.36, p=0.10$ ) in right colonic tumors. ${ }^{25}$ These data, in line with FIRE-3, suggest an advantage for incorporating anti-EGFR therapy over bevacizumab in left colonic metastatic cancers while significant concerns arise from a potential harm, or at least, lack of benefit from anti-EGFR therapy in the first-line treatment of right colonic metastatic cancer.

\section{Conclusions}

Retrospective analysis of multiple randomized phase III clinical trials point to the effectiveness of anti-EGFR therapy across lines of treatment in RAS wild-type metastatic colorectal cancer. The significant improvements in OS across FIRE-3 and CALGB 80405 in the first-line setting in left colon cancers suggest that this biologic class of agents should be the favorite choice when considering targeted therapy in this left colon cancer patients (Figure 1). BRAF-mutated colon cancers, while representing a very small portion of left colon cancers, are not known to benefit from anti-EGFR therapies and can be considered for alternative strategies, including folinic acid, fluorouracil, oxaliplatin, irinotecan, plus bevacizumab (FOLFOXIRIBV). ${ }^{26}$ Of note, the current evidence-based data apply to doublet combination chemotherapy backbones. While anti-EGFR therapies appear to be superior to bevacizumab when combined with FOLFOX or FOLFIRI, we have no comparative data for FOLFOX plus panitumumab or FOLFIRI plus cetuximab to FOLFOXIRI-BV. Given the significant improvements noted with FOLFOXIRI-BV over FOLFIRI-BV across different molecular subgroups of metastatic colorectal cancer, FOLFOXIRI-BV remains an appropriate choice for left (and right) colonic RAS wild-type metastatic colorectal cancer ${ }^{26}$ Finally, some left colon cancer patients may not find the potential side effects of anti-EGFR therapy palatable in a first-line setting. In those patients, a bevacizumab-based backbone therapy is appropriate, irrespective of chemotherapy backbone.

The current evidence suggests that RAS wild-type right colonic tumors should not receive anti-EGFR therapy in the first- and second-line settings 
(Figure 1). The exclusion of BRAF mutations from this subgroup does not unearth a clinical benefit in the RAS/BRAF wild-type patients. ${ }^{21}$ While a survival advantage is missing with anti-EGFR in front-line treatment settings, one must acknowledge the limitations of this evidence given the small right colonic data sets. The presence of a small trend toward improvement in OS with cetuximab monotherapy in KRAS wild-type right colonic tumors as well as the trends in improved RR on the CRYSTAL and PRIME in right colonic tumors, suggest a potential benefit of anti-
EGFR in a small subgroup of right colonic tumors. Defining additional biomarkers of response to anti-EGFR therapy in right colonic patients will be important to optimize our personalized treatments with antiEGFR therapy. Until then, anti-EGFR therapies should be avoided in firstand second-line therapies. However, until we have further conclusive evidence in chemorefractory settings, I am not ready to completely exclude this therapeutic option in our chemorefractory RAS/BRAF wildtype colonic patients.
1. Douillard JY, Siena S, Cassidy J, et al., Randomized, phase III trial of panitumumab with infusional fluorouracil, leucovorin, and oxaliplatin (FOLFOX4) verSUS FOLFOX4 alone as first-line treatment in patients with previously untreated metastatic colorectal cancer: the PRIME study I Clin Oncol, 2010:28:4697-705.

2. Douillard JY, Oliner KS, siena S, et al., Panitumumab-FOLFOX4 treatment and RAS mutations in colorectal cancer, N Eng/ J Med 2013;369:1023-34.

3. Van Cutsem $\mathrm{E}$, Kohne $\mathrm{CH}$, Lang I, et al., Cetuximab plus irinotecan, fluorouracil, and leucovorin as first-line treatment for metastatic colorectal cancer: updated analysis of overall surviva mecording to tumor KRAS and BRAF mutation status, J Clin Oncol, 2011:29:2011-9.

4. Van Cutsem E, Lenz HJ, Kohne $\mathrm{CH}$, et al., Fluorouracil, leucovorin, and irinotecan plus cetuximab treatment and RAS mutations in colorectal cancer, J Clin Oncol, 2015;33:692-700.

5. Peeters $M$, Oliner KS, Price TJ, et al., Analysis of KRAS/NRAS mutations in a phase III study of Panitumumab with FOLFIR compared with FOLFIRI alone as second-line treatment for metastatic colorectal cancer, Clin Cancer Res, 2015;21:5469-79.

6. Gong J, Cho M, Fakih M, RAS and BRAF in metastatic colorectal cancer management, J Gastrointest Oncol, 2016;7:687-704.

7. Distler P, Holt PR, Are right- and left-sided colon neoplasms distinct tumors?, Dig Dis, 1997;15:302-11.

8. Glebov OK, Rodriguez LM, Nakahara K, et al., Distinguishing right from left colon by the pattern of gene expression, Cancer Epidemiol Biomarkers Prev, 2003:12:755-62.

9. Missiaglia E, Jacobs B, D'Ario G, et al., Distal and proximal colon cancers differ in terms of molecular, pathological, and clinical features, Ann Oncol, 2014:25:1995-2001.

10. Meguid RA, Slidell MB, Wolfgang $C L$, et al., Is there a difference in survival between right- versus left-sided colon cancers? , Ann Surg Oncol, 2008;15:2388-94.
11. Weiss JM, Pfau PR, O'Connor ES, et al., Mortality by stage for right- versus left-sided colon cancer: analysis of surveillance epidemiology, and end results-Medicare data, I Clin Oncol, 2011:29:4401-9.

12. Benedix F, Kube R, Meyer F, et al., Comparison of 17,641 patients with right- and left-sided colon cancer: differences in epidemiology, perioperative course, histology, and survival, Dis Colon Rectum, 2010;53:57-64.

13. Warschkow R, Sulz MC, Marti L, et al., Better survival in right-sided versus left-sided stage I-III colon cancer patients, BMC Cancer 2016;16:554.

14. Zhang $\mathrm{Y}$, Ma J, Zhang S, et al., A prognostic analysis of 895 cases of stage III colon cancer in different colon subsites, Int I colorecta Dis, 2015;30:1173-83.

15. Kerr DJ, Domingo E, Kerr R, Is sidedness prognostically important across all stages of colorectal cancer?, Lancet Oncol, 2016;17:1480-2.

16. Holch JW, Ricard I, Stintzing S, et al., The relevance of primary tumour location in patients with metastatic colorectal cancer. a meta-analysis of first-line clinical trials, Eur J Cancer, 2017:70:87-98.

17. Moretto R, Cremolini $C$, Rossini $D$, et al., Location of primary tumor and benefit from anti-epidermal growth factor receptor monoclonal antibodies in patients with RAS and BRAF wild-typ metastatic colorectal cancer, Oncologist, 2016;21:988-94.

18. De Roock W, Claes B, Bernasconi D, et al., Effects of KRAS, BRAF, NRAS, and PIK3CA mutations on the efficacy of cetuximab plus chemotherapy in chemotherapy-refractory metastatic colorecta cancer: a retrospective consortium analysis, Lancet Oncol, 2010;11:753-62.

19. Brule SY, Jonker DJ, Karapetis CS, et al., Location of colon cancer (right-sided versus left-sided) as a prognostic factor and a predictor of benefit from cetuximab in NCIC C0.17, Eur $J$ Cancer 2015;51:1405-14.
20. Wang F, Bai L, Liu TS, et al., Right-sided colon cancer and left-sided colorectal cancers respond differently to cetuximab, Chin J Cancer, 2015;34:384-93

21. Tejpar S, Stintzing S, Ciardiello F, et al., Prognostic and predictive relevance of primary tumor location in patients with RAS wild-type metastatic colorectal cancer. retrospective analyses of the CRYSTAL and FIRE-3 trials, JAMA OnCol, 2017;3:194-201.

22. BoeckX N, Toler A, Op de Beeck K, et al., Primary tumor sidedness impacts on prognosis and treatment outcome: results from three randomized studies of panitumumab plus chemotherapy versus chemotherapy plus panitumumab, Ann Oncol, 2016;27:15-42.

23. Heinemann $V$ von Weikersthal LF Decker T, et al., FOLFIRI plus cetuximab versus FOLFIRI plus bevacizumab as first-line treatment for patients with metastatic colorectal cance (FIRE-3): a randomised, open-label, phase 3 trial, Lancet Oncol, 2014;15:1065-75.

24. Venook A, Niedzwiecki D, Lenz H, et al., CALGB/SWOG 80405: phase III trial of irinotecan/5-FU/leucovorin (FOLFIRI) or oxaliplatin/5-FU/leucovorin (MFOLFOX6) with bevacizumab (BV) or cetuximab (CET) for patients (pts) with KRAS wild-type (wt) or cetuximab (CET) for patients (pts) With KRAS wild-type (Wt) untreated metastatic adenocarcinoma of the col
(MCRC), I Clin Oncol, 2014;32(Suppl):abst LBA4.

25. Venook A, Niedzwiecki D, Innocenti F, et al., Impact of primary ${ }^{\left(1{ }^{\circ}\right)}$ tumor location on overall survival (OS) and progression-free survival (PFS) in patients (pts) with metastatic colorectal cancer (mCRC): analysis of CALGB/SWOG 80405 (Alliance), J Clin Oncol 2016;34(Suppl):abstr 3504.

26. Cremolini C, Loupakis F, Antoniotti C, et al., FOLFOXIRI plus bevacizumab versus FOLFIRI plus bevacizumab as first-line treatment of patients with metastatic colorectal cancer. updated overall survival and molecular subgroup analyses of the open-label, phase 3 TRIBE study, Lancet Oncol, 2015;16:1306-15. 